\title{
Additive problems involving primes of special type
}

\author{
by \\ Yingchun Cai and MingGao Lu (Shanghai)
}

1. Introduction. In 1742, in his letters to Euler, Goldbach proposed his well-known conjectures, which can be formulated in modern mathematical terms as follows:

(A) For any even integer $n \geq 4$, the equation

$$
n=p_{1}+p_{2}
$$

is solvable in primes $p_{1}, p_{2}$.

(B) For any odd integer $n \geq 7$, the equation

$$
n=p_{1}+p_{2}+p_{3}
$$

is solvable in primes $p_{1}, p_{2}, p_{3}$.

Nowadays the best results concerning Conjectures (A) and (B) are due to Chen [2] and Vinogradov [18] respectively. In 1937 Vinogradov [18] showed that Conjecture (B) holds for any sufficiently large odd integers. As for Conjecture (A), in 1973, by adding his ingenious innovations into sieve theory, Chen [2] proved that any sufficiently large even integer $n$ can be represented in the form

$$
n=p_{1}+P_{2}
$$

where $p_{1}$ is a prime and $P_{2}$ is an almost-prime with at most two prime factors.

In 1938, basing upon Vinogradov's work, Hua [9] showed that for sufficiently large $n \equiv 5(\bmod 24)$, the equation

$$
n=p_{1}^{2}+p_{2}^{2}+p_{3}^{2}+p_{4}^{2}+p_{5}^{2}
$$

is solvable in primes $p_{1}, p_{2}, p_{3}, p_{4}, p_{5}$.

In 1939, by Vinogradov's method, van der Corput [17] proved that there exist infinitely many arithmetic progressions of three different prime terms. In 1981, Heath-Brown [8] showed that there exist infinitely many arithmetic 
progressions of four different terms, three of which are primes, and the fourth is $P_{2}$. In 2006, Green and Tao [3] established that there exist infinitely many arithmetic progressions consisting of three different primes $p_{1}<p_{2}<p_{3}$ such that $p_{j}+2=P_{2}$ for each $j=1,2,3$. Recently [4] they showed that this holds for any number $k \geq 3$ of primes.

Motivated by Heath-Brown [8], Tolev [14-16] and Peneva [12, 13] studied additive problems with primes $p$ such that $p+2$ is an almost-prime. In [16] Tolev showed, by using the vector sieve developed in [1], that:

$1)$ If $n$ is sufficiently large and $n \equiv 3(\bmod 6)$, then the equation $(1.2)$ is solvable in primes $p_{1}, p_{2}, p_{3}$ such that

$$
p_{1}+2=P_{2}, \quad p_{2}+2=P_{5}, \quad p_{3}+2=P_{7} .
$$

$2)$ If $n$ is sufficiently large and $n \equiv 5(\bmod 24)$, then the equation (1.4) is solvable in primes $p_{1}, p_{2}, p_{3}, p_{4}, p_{5}$ such that

$$
p_{1}+2=P_{2}, \quad p_{2}+2=P_{2}^{\prime}, \quad p_{3}+2=P_{5}, \quad p_{4}+2=P_{5}^{\prime}, \quad p_{5}+2=P_{8} .
$$

In this paper, by inserting a weighted sieve approach into Tolev's argument, we obtain the following sharper results

TheOREM 1. If $n$ is sufficiently large and $n \equiv 5(\bmod 24)$, then the equation (1.4) is solvable in primes $p_{1}, p_{2}, p_{3}, p_{4}, p_{5}$ such that

$$
p_{1}+2=P_{2}, \quad p_{2}+2=P_{2}^{\prime}, \quad p_{3}+2=P_{4}, \quad p_{4}+2=P_{4}^{\prime}, \quad p_{5}+2=P_{5} .
$$

Theorem 2. If $n$ is sufficiently large and $n \equiv 3(\bmod 6)$, then the equation (1.2) is solvable in primes $p_{1}, p_{2}, p_{3}$ such that

$$
p_{1}+2=P_{2}, \quad p_{2}+2=P_{3}, \quad p_{3}+2=P_{5} .
$$

Theorem $2^{\prime}$. If $n$ is sufficiently large and $n \equiv 3(\bmod 6)$, then the equation (1.2) is solvable in primes $p_{1}, p_{2}, p_{3}$ such that

$$
p_{1}+2=P_{2}, \quad p_{2}+2=P_{4}, \quad p_{3}+2=P_{4}^{\prime} .
$$

2. Some preliminary lemmas. In this paper we follow the notation of Tolev [16] as closely as possible. For the convenience of the reader, we recall some of it here.

Let $P_{r}$ denote an almost-prime with at most $r$ prime factors, counted according to multiplicity. Let $A \geq 10^{4}$ denote a constant. The constants in $O$-terms and «-symbols are absolute or depend only on $A$. Let $N$ denote a sufficiently large integer and $X=N^{1 / 2}, Q=(\log X)^{10^{3} A}$. The letter $p$, with or without subscripts, is reserved for primes. Boldface letters denote vectors of dimension three. As usual, $\mu(n), \varphi(n), \tau(n), \nu_{2}(n)$ denote the Möbius function, Euler's function, the number of divisors of $n$ and the total number of prime factors of $n$ respectively, and $\tau_{k}(n)$ denotes the number of solutions of the equation $m_{1} \cdots m_{k}=n$ in positive integers $m_{1}, \ldots, m_{k}$, 
$\tau_{2}(n)=\tau(n)$. By $\left(m_{1}, \ldots, m_{k}\right)$ we denote the largest common divisor of $m_{1}, \ldots, m_{k}$. If $p^{l} \mid m$ but $p^{l+1} \nmid m$ then we write $p^{l} \| m$. We use $e(\alpha)$ to denote $e^{2 \pi i \alpha}$ and $e_{q}(\alpha)=e(\alpha / q)$. We denote by $\sum_{x(q)}$ and $\sum_{x(q) *}$ sums with $x$ running over a complete system and a reduced system of residues modulo $q$ respectively. By $\left(\frac{l}{p}\right)$ we denote the Legendre symbol. We use $\mathbb{N}$ to denote the set of positive integers. For $\mathbf{k}=\left\{k_{1}, k_{2}, k_{3}\right\} \in \mathbb{N}^{3}$ and $\mathbf{l}=\left\{l_{1}, l_{2}, l_{3}\right\} \in \mathbb{N}^{3}$, define $\mathbf{k l}=\left\{k_{1} l_{1}, k_{2} l_{2}, k_{3} l_{3}\right\}$. For an arithmetic function $f$ we define $f(\mathbf{k})=$ $f\left(k_{1}\right) f\left(k_{2}\right) f\left(k_{3}\right)$. For a set $S$, we denote its cardinality by $|S|$. Set

$$
\begin{aligned}
& S_{k}(q, a)=\frac{\varphi((k, q))}{\varphi(q)} \sum_{\substack{x(q) * \\
x+2 \equiv 0(\bmod (k, q))}} e_{q}\left(a x^{2}\right), \\
& S_{\mathbf{k}}(q, a)=\prod_{j=1}^{3} S_{k_{j}}(q, a), \quad \mathbf{k}=\left\{k_{1}, k_{2}, k_{3}\right\} \in \mathbb{N}^{3}, \\
& t(q ; n ; \mathbf{k})=\sum_{a(q) *} S_{\mathbf{k}}(q, a) e_{q}(-a n), \\
& \mathfrak{S}(n ; Q ; \mathbf{k})=8 \prod_{3 \leq p<Q}(1+t(p ; n ; \mathbf{k})), \\
& I(n ; \mathbf{k})=\sum_{\substack{p_{1}^{2}+p_{2}^{2}+p_{3}^{2}=n \\
p_{j}+2 \equiv 0\left(\bmod k_{j}\right) \\
j=1,2,3}} \log \mathbf{p} \\
& \mathfrak{R}(n ; Q ; \mathbf{k})=I(n ; \mathbf{k})-\frac{\pi}{4} n^{1 / 2} \frac{\mathfrak{S}(n ; Q ; \mathbf{k})}{\varphi(\mathbf{k})}, \\
& h_{0}(p)= \begin{cases}\frac{\left(\frac{-n}{p}\right) p^{2}+\left(3\left(\frac{n}{p}\right)+3\left(\frac{-1}{p}\right)\right) p+1}{(p-1)^{3}}, & p \nmid n, \\
\frac{-3\left(\frac{-1}{p}\right) p-1}{(p-1)^{2}}, & p \mid n,\end{cases} \\
& h_{1}(p)= \begin{cases}\frac{\left(-2\left(\frac{n-4}{p}\right)-\left(\frac{-1}{p}\right)\right) p-1}{(p-1)^{2}}, & p \nmid(n-4), \\
\frac{\left(\frac{-1}{p}\right) p+1}{p-1}, & p \mid(n-4),\end{cases} \\
& h_{2}(p)= \begin{cases}\frac{\left(\frac{n-8}{p}\right) p+1}{p-1}, & p \nmid(n-8), \\
-1, & p \mid(n-8),\end{cases} \\
& h_{3}(p)= \begin{cases}-1, & p \nmid(n-12), \\
p-1, & p \mid(n-12) .\end{cases}
\end{aligned}
$$


Lemma 1 ([16]). For $\mathbf{k} \in \mathbb{N}^{3}$ with square-free odd components, the function $t(q ; n ; \mathbf{k})$ is multiplicative with respect to $q$. We have

$$
t\left(2^{l} ; n ; \mathbf{k}\right)= \begin{cases}1, & l=1 \\ 2, & l=2 \\ 3, & l=3 \\ 0, & l>3\end{cases}
$$

For $p>2$ we have

$$
t\left(p^{l} ; n ; \mathbf{k}\right)= \begin{cases}h_{j}(p), & p^{j} \| k_{1} k_{2} k_{3} \text { and } l=1, \\ 0, & l>1 .\end{cases}
$$

Lemma 2 ([16]). Put

$$
K_{1}=K_{2}=X^{1 / 2}(\log X)^{-2 \cdot 10^{4} A}, \quad K_{3}=X^{1 / 3}(\log X)^{-2 \cdot 10^{4} A}
$$

and let $\beta_{j}(k), j=1,2,3$, denote complex numbers such that

$$
\begin{aligned}
& \beta_{j}(k)=0 \quad \text { if } 2 \mid k \text { or } \mu(k)=0 \text { or } k>K_{j}, \\
& \left|\beta_{j}(k)\right| \leq \tau_{3}(k) .
\end{aligned}
$$

Then

$$
\sum_{n}^{*}\left|\sum_{\substack{k_{j} \leq K_{j} \\ j=1,2,3}} \beta_{1}\left(k_{1}\right) \beta_{2}\left(k_{2}\right) \beta_{3}\left(k_{3}\right) \Re(n ; Q ; \mathbf{k})\right| \ll X^{3} \log ^{-A} X,
$$

where $\sum_{n}^{*}$ means that the summation is taken over the integers $n$ satisfying

$$
N / 2 \leq n \leq N, \quad n \equiv 3(\bmod 24) \text { and } n \not \equiv 0(\bmod 5) .
$$

Lemma 3 ([12]). Suppose that $\phi\left(n_{1}, n_{2}, n_{3}\right)$ is a function defined on $\mathbb{N}^{3}$ such that for any $\left\{n_{1}, n_{2}, n_{3}\right\},\left\{l_{1}, l_{2}, l_{3}\right\} \in \mathbb{N}^{3}$ satisfying $\left(n_{1} n_{2} n_{3}, l_{1} l_{2} l_{3}\right)=1$ we have $\phi\left(n_{1} l_{1}, n_{2} l_{2}, n_{3} l_{3}\right)=\phi\left(n_{1}, n_{2}, n_{3}\right) \phi\left(l_{1}, l_{2}, l_{3}\right)$. Then the function

$$
\Phi(n)=\sum_{d_{1}, d_{2}, d_{3} \mid n} \phi\left(d_{1}, d_{2}, d_{3}\right)
$$

is multiplicative.

For fixed $D \geq 1$ we define Rosser's weights $\lambda^{ \pm}(d)$ of order $D$ as follows: for $d=p_{1} \cdots p_{r}$ with $p_{1}>\cdots>p_{r}$, let

$$
\begin{aligned}
& \lambda^{+}(d)= \begin{cases}(-1)^{r} & \text { if } p_{1} \cdots p_{2 l} p_{2 l+1}^{3}<D \text { whenever } 0 \leq l \leq(r-1) / 2, \\
0 & \text { otherwise, }\end{cases} \\
& \lambda^{-}(d)= \begin{cases}(-1)^{r} & \text { if } p_{1} \cdots p_{2 l} p_{2 l}^{3}<D \text { whenever } 1 \leq l \leq r / 2, \\
0 & \text { otherwise. }\end{cases}
\end{aligned}
$$

Finally, put $\lambda^{ \pm}(1)=1$ and $\lambda^{ \pm}(d)=0$ if $d$ is not square-free. 
Lemma $4([10,11])$. Let $\mathscr{P}$ denote a set of primes and set

$$
P(z)=\prod_{\substack{p<z \\ p \in \mathscr{P}}} p
$$

Then for Rosser's weights $\lambda^{ \pm}(d)$ of order $D$, any integer $n \geq 1$ and real number $z \geq 2$ we have

$$
\sum_{d \mid(n, P(z))} \lambda^{-}(d) \leq \sum_{d \mid(n, P(z))} \mu(d) \leq \sum_{d \mid(n, P(z))} \lambda^{+}(d) .
$$

Moreover, for any multiplicative function $\omega$ satisfying

$$
\begin{cases}0<\omega(p)<p & \text { if } p \in \mathscr{P}, \\ \omega(p)=0 & \text { if } p \notin \mathscr{P},\end{cases}
$$

and

$$
\prod_{w_{1} \leq p<w_{2}}\left(1-\frac{\omega(p)}{p}\right)^{-1} \leq \frac{\log w_{2}}{\log w_{1}}\left(1+\frac{L}{\log w_{1}}\right)
$$

(for all $2 \leq w_{1}<w_{2}$, where $L$ is a positive constant), we have

$$
V(z) \geq \sum_{d \mid P(z)} \lambda^{-}(d) \frac{\omega(d)}{d} \geq V(z)\left(f(s)+O\left(e^{\sqrt{L}-s} \log ^{-1 / 3} D\right)\right)
$$

for $2 \leq z \leq D^{1 / 2}$, and

$$
V(z) \leq \sum_{d \mid P(z)} \lambda^{+}(d) \frac{\omega(d)}{d} \leq V(z)\left(F(s)+O\left(e^{\sqrt{L}-s} \log ^{-1 / 3} D\right)\right)
$$

for $2 \leq z \leq D$, where

$$
V(z)=\prod_{p<z}\left(1-\frac{\omega(p)}{p}\right), \quad s=\frac{\log D}{\log z},
$$

and $f(s)$ and $F(s)$ denote the classical functions in the linear sieve.

Lemma $5([5,6])$. For the functions $f(s)$ and $F(s)$ we have

$$
\begin{aligned}
& s f(s)=2 e^{\gamma} \log (s-1), \quad 2 \leq s \leq 4 ; \\
& s f(s)=2 e^{\gamma}\left(\log (s-1)+\int_{2}^{s-2} \frac{\log (t-1)}{t} \log \frac{s-1}{t+1} d t\right), \quad 4 \leq s \leq 6 ; \\
& s F(s)=2 e^{\gamma}, \quad 1 \leq s \leq 3 ; \\
& s F(s)=2 e^{\gamma}\left(1+\int_{2}^{s-1} \frac{\log (t-1)}{t} d t\right), \quad 3 \leq s \leq 5 ;
\end{aligned}
$$




$$
\begin{aligned}
s F(s)= & 2 e^{\gamma}\left(1+\int_{2}^{s-1} \frac{\log (t-1)}{t} d t\right. \\
& \left.+\int_{2}^{s-3} \frac{\log (t-1)}{t} d t \int_{t+2}^{s-1} \log \frac{u-1}{t+1} \frac{d u}{u}\right), \quad 5 \leq s \leq 7,
\end{aligned}
$$

where $\gamma=0.577 \ldots$ denotes Euler's constant.

3. Some propositions. The following propositions play a central role in the proof of the theorems.

Proposition 1. Denote by $\mathcal{K}$ the set of integers $n$ for which the equation $n=p_{1}^{2}+p_{2}^{2}+p_{3}^{2}$ is solvable in primes $p_{1}, p_{2}, p_{3}$ such that

$$
p_{1}+2=P_{4}, \quad p_{2}+2=P_{4}^{\prime}, \quad p_{3}+2=P_{5},
$$

and set

$$
\mathcal{F}=\{N / 2 \leq n \leq N: n \equiv 3(\bmod 24), n \not \equiv 0(\bmod 5)\} \backslash \mathcal{K} .
$$

Let $\mathcal{Y}(N)$ denote the cardinality of $\mathcal{F}$. Then for any $B>0$ we have

$$
\mathcal{Y}(N) \ll N \log ^{-B} N \text {. }
$$

Proposition 2. Denote by $\mathcal{K}_{0}$ the set of integers $n$ for which the equation $n=p_{1}+p_{2}$ is solvable in primes $p_{1}, p_{2}$ such that

$$
p_{1}+2=P_{3}, \quad p_{2}+2=P_{5},
$$

and set

$$
\mathcal{F}_{0}=\{N / 2 \leq n \leq N: n \equiv 4(\bmod 6)\} \backslash \mathcal{K}_{0} .
$$

Let $\mathcal{Y}_{0}(N)$ denote the cardinality of $\mathcal{F}_{0}$. Then for any $B>0$ we have

$$
\mathcal{Y}_{0}(N) \ll N \log ^{-B} N \text {. }
$$

Proposition 2'. Denote by $\mathcal{K}_{1}$ the set of integers $n$ for which the equation $n=p_{1}+p_{2}$ is solvable in primes $p_{1}, p_{2}$ such that

$$
p_{1}+2=P_{4}, \quad p_{2}+2=P_{4}^{\prime},
$$

and set

$$
\mathcal{F}_{1}=\{N / 2 \leq n \leq N: n \equiv 4(\bmod 6)\} \backslash \mathcal{K}_{1} .
$$

Let $\mathcal{Y}_{1}(N)$ denote the cardinality of $\mathcal{F}_{1}$. Then for any $B>0$ we have

$$
\mathcal{Y}_{1}(N) \ll N \log ^{-B} N \text {. }
$$

4. Proof of the propositions. In this paper we present only the proof of Proposition 1. By the Proposition in [15] and similar arguments, Propositions 2 and $2^{\prime}$ follow easily. In the proof of Proposition 1 we adopt the 
following notation:

$$
\begin{aligned}
& Q_{0}=\log ^{3 / 5} X, \quad D_{0}=\exp \left(\log ^{3 / 5} X\right), \\
& D_{1}=D_{2}=X^{1 / 2} \exp \left(-4 \log ^{3 / 5} X\right), \quad D_{3}=X^{1 / 3} \exp \left(-4 \log ^{3 / 5} X\right), \\
& w_{1}=w_{2}=D_{1}^{1 / 5}, \quad w_{3}=D_{3}^{1 / 6}, \quad z_{1}=z_{2}=D_{1}^{4 / 5}, \quad z_{3}=D_{3}^{5 / 6}, \\
& \theta_{1}=\theta_{2}=\frac{1}{2.498}, \quad \theta_{3}=\frac{1}{2.398}, \quad s_{1}=s_{2}=5, \quad s_{3}=6, \\
& \mathfrak{R}=\{p: p \geq 11, p \nmid(n-4)\} \cup\{p: p \geq 11, p \mid(n-4), p \equiv 1(\bmod 4)\}, \\
& \mathcal{B}_{0}=\prod_{3 \leq p<Q_{0}} p, \quad \mathcal{P}_{0}=\prod_{\substack{Q_{0} \leq p<Q \\
p \in \mathfrak{R}}} p, \\
& \mathcal{P}_{j}=\prod_{Q \leq p<w_{j}} p, \quad Q_{j}=\mathcal{B}_{0} \mathcal{P}_{0} \mathcal{P}_{j}, \quad P\left(w_{j}\right)=\prod_{p<w_{j}} p, \quad j=1,2,3, \\
& g_{j}^{\prime}(x)=1-\frac{\log x}{\log z_{j}}, \quad g_{j}(x)=\sum_{\substack{w_{j} \leq p<z_{j} \\
p \mid x}} g_{j}^{\prime}(p), \quad j=1,2,3,
\end{aligned}
$$

$\lambda_{j}^{ \pm}(d)$ Rosser's weights of order $D_{j}, \quad j=0,1,2,3$,

$\lambda_{j}^{ \pm(p)}(d)$ Rosser's weights of order $D_{j} / p, \quad w_{j} \leq p<z_{j}, j=1,2,3$,

$$
\begin{aligned}
\Phi_{j} & =\sum_{k \mid\left(p_{j}+2, \mathcal{B}_{0}\right)} \mu(k), \quad \Psi_{j}=\sum_{l \mid\left(p_{j}+2, \mathcal{P}_{0}\right)} \mu(l), \quad \Lambda_{j}=\sum_{m \mid\left(p_{j}+2, \mathcal{P}_{j}\right)} \mu(m), \\
\Psi_{j}^{ \pm} & =\sum_{k \mid\left(p_{j}+2, \mathcal{P}_{0}\right)} \lambda_{0}^{ \pm}(k), \quad \Lambda_{j}^{ \pm}=\sum_{l \mid\left(p_{j}+2, \mathcal{P}_{j}\right)} \lambda_{j}^{ \pm}(l), \quad j=1,2,3, \\
\mathcal{F}^{*} & =\left\{n: n \in \mathcal{F}, \nu_{2}(n-4) \leq A \log \log X\right\} .
\end{aligned}
$$

For the proof of Proposition 1 we consider the sum

$$
\begin{aligned}
\Gamma & =\sum_{n \in \mathcal{F}^{*}} \sum_{\begin{array}{r}
p_{1}^{2}+p_{2}^{2}+p_{3}^{2}=n \\
\left(p_{j}+2, Q_{j}\right)=1 \\
j=1,2,3
\end{array}}(\log \mathbf{p})\left(1-\sum_{j=1}^{3} \theta_{j} g_{j}\left(p_{j}+2\right)\right) \\
& =\sum_{n \in \mathcal{F}^{*}} \sum_{\substack{\left.p_{1}^{2}+p_{2}^{2}+p_{3}^{2}=n \\
p_{j}+2, Q_{j}\right)=1 \\
j=1,2,3}} \log \mathbf{p}-\sum_{j=1}^{3} \theta_{j} \sum_{\substack{n \in \mathcal{F}^{*} \\
p_{1}^{2}+p_{2}^{2}+p_{3}^{2}=n \\
\left(p_{j}+2, Q_{j}=1 \\
j=1,2,3\right.}}(\log \mathbf{p}) g_{j}\left(p_{j}+2\right) \\
& =\Gamma^{(0)}-\sum_{j=1}^{3} \theta_{j} \Gamma_{j}^{(1)}=\Gamma^{(0)}-\Gamma^{(1)} .
\end{aligned}
$$


A) The upper bound for $\Gamma$. Write

$$
\Gamma=\sum_{n \in \mathcal{F}^{*}} w(n), \quad w(n)=\sum_{\substack{\left.p_{1}^{2}+p_{2}^{2}+p_{3}^{2}=n \\ p_{j}+2, Q_{j}\right)=1 \\ j=1,2,3}}(\log \mathbf{p})\left(1-\sum_{j=1}^{3} \theta_{j} g_{j}\left(p_{j}+2\right)\right) .
$$

Let $n \in \mathcal{F}^{*}$ give a positive contribution to $\Gamma$. Then we have

$$
\begin{aligned}
& p_{1}^{2}+p_{2}^{2}+p_{3}^{2}=n, \\
& \left(p_{j}+2, Q_{j}\right)=1, \quad j=1,2,3, \\
& \theta_{j} g_{j}\left(p_{j}+2\right)<1, \quad j=1,2,3,
\end{aligned}
$$

for some primes $p_{1}, p_{2}, p_{3}$.

The contribution from those representations satisfying (4.2)-(4.4) with some $p_{j}+2$ non-square-free is

$$
\begin{aligned}
& \ll \sum_{w_{3} \leq p<X^{1 / 2}} \sum_{\substack{p_{3} \leq X \\
p_{3} \equiv-2\left(\bmod p^{2}\right)}} \sum_{p_{1}^{2}+p_{2}^{2} \leq N-p_{3}^{2}} \log ^{3} X \\
& \ll N \sum_{w_{3} \leq p<X^{1 / 2}} \sum_{\substack{p_{3} \leq X \\
p_{3} \equiv-2\left(\bmod p^{2}\right)}} \log ^{3} X \\
& \ll N \sum_{w_{3} \leq p<X^{1 / 2}}\left(\frac{X}{p^{2}}+1\right) \log ^{3} X \\
& \ll\left(X^{3} w_{3}^{-1}+X^{5 / 2}\right) \log ^{3} X \ll X^{59 / 20} .
\end{aligned}
$$

For the remaining representations satisfying (4.2)-(4.4), $p_{j}+2$ is squarefree for $j=1,2,3$. If $\left(p_{j}+2, P\left(w_{j}\right)\right)=1$ for $j=1,2,3$, then we have

$$
\nu_{2}\left(p_{j}+2\right)=\sum_{\substack{p \mid\left(p_{j}+2\right) \\ p \geq w_{j}}} 1, \quad j=1,2,3 .
$$

By (4.4) we have

$$
\begin{aligned}
& \sum_{\substack{p \mid\left(p_{j}+2\right) \\
w_{j} \leq p<z_{j}}}\left(1-\frac{\log p}{\log z_{j}}\right)<\frac{1}{\theta_{j}}, \quad j=1,2,3, \\
& \sum_{\substack{p \mid\left(p_{j}+2\right) \\
p \geq w_{j}}}\left(1-\frac{\log p}{\log z_{j}}\right)<\frac{1}{\theta_{j}}, \quad j=1,2,3, \\
& \sum_{\substack{p \mid\left(p_{j}+2\right) \\
p \geq w_{j}}} 1<\frac{1}{\theta_{j}}+\frac{\log \left(p_{j}+2\right)}{\log z_{j}}, \quad j=1,2,3 .
\end{aligned}
$$


From (4.6)-(4.7) we get

$$
\nu_{2}\left(p_{j}+2\right) \leq \begin{cases}4, & j=1,2, \\ 5, & j=3\end{cases}
$$

Now (4.2) and (4.8) contradict the fact that $n \in \mathcal{F}^{*}$, so we must have $\left(p_{j}+2, P\left(w_{j}\right)\right)>1$ for some $j$. Without loss of generality we assume that

$$
\left(p_{1}+2, P\left(w_{1}\right)\right)>1 \text {. }
$$

If $p_{1}=2$ then

$$
w(n) \leq \sum_{m_{1}^{2}+m_{2}^{2}+4=n} \log ^{3} X .
$$

If $p_{1}>2$ then from (4.3) and (4.9) we deduce that $p_{1}+2$ has a prime factor $p>2$ such that $p \mid(n-4)$ and $p \equiv 3(\bmod 4)$. Hence $p_{2}^{2}+p_{3}^{2} \equiv 0(\bmod p)$, which implies that $p_{2}=p_{3}=p$, and we have

$$
w(n) \leq \sum_{p \mid(n-4)} \log ^{3} X .
$$

From (4.5) and (4.10)-(4.11) we obtain

$$
\begin{aligned}
\Gamma & \ll X^{59 / 20}+\left(\sum_{m_{1}^{2}+m_{2}^{2}+4 \leq N} 1+\sum_{n \leq N} \tau(n-4)\right) \log ^{3} X \\
& \ll X^{59 / 20}+X^{2} \log ^{4} X \ll X^{59 / 20} .
\end{aligned}
$$

B) The lower bound for $\Gamma$. In this part we give a lower bound for $\Gamma$ by applying the vector sieve in [1].

- The lower bound for $\Gamma^{(0)}$. By (2.1) and the inequality

$$
\begin{aligned}
\Psi_{1} \Psi_{2} \Psi_{3} \Lambda_{1} \Lambda_{2} \Lambda_{3} \geq & \Psi_{1}^{-} \Psi_{2}^{+} \Psi_{3}^{+} \Lambda_{1}^{+} \Lambda_{2}^{+} \Lambda_{3}^{+}+\Psi_{1}^{+} \Psi_{2}^{-} \Psi_{3}^{+} \Lambda_{1}^{+} \Lambda_{2}^{+} \Lambda_{3}^{+} \\
& +\Psi_{1}^{+} \Psi_{2}^{+} \Psi_{3}^{-} \Lambda_{1}^{+} \Lambda_{2}^{+} \Lambda_{3}^{+}+\Psi_{1}^{+} \Psi_{2}^{+} \Psi_{3}^{+} \Lambda_{1}^{-} \Lambda_{2}^{+} \Lambda_{3}^{+} \\
& +\Psi_{1}^{+} \Psi_{2}^{+} \Psi_{3}^{+} \Lambda_{1}^{+} \Lambda_{2}^{-} \Lambda_{3}^{+}+\Psi_{1}^{+} \Psi_{2}^{+} \Psi_{3}^{+} \Lambda_{1}^{+} \Lambda_{2}^{+} \Lambda_{3}^{-} \\
& -5 \Psi_{1}^{+} \Psi_{2}^{+} \Psi_{3}^{+} \Lambda_{1}^{+} \Lambda_{2}^{+} \Lambda_{3}^{+}
\end{aligned}
$$

of [16], we get

$$
\begin{aligned}
\Gamma^{(0)} & =\sum_{n \in \mathcal{F}^{*}} \sum_{p_{1}^{2}+p_{2}^{2}+p_{3}^{2}=n}(\log \mathbf{p}) \Phi_{1} \Phi_{2} \Phi_{3} \Psi_{1} \Psi_{2} \Psi_{3} \Lambda_{1} \Lambda_{2} \Lambda_{3} \\
& \geq \sum_{j=1}^{6} \Gamma_{j}^{(0)}-5 \Gamma_{7}^{(0)},
\end{aligned}
$$

where

$$
\Gamma_{1}^{(0)}=\sum_{n \in \mathcal{F}^{*}} \sum_{p_{1}^{2}+p_{2}^{2}+p_{3}^{2}=n}(\log \mathbf{p}) \Phi_{1} \Phi_{2} \Phi_{3} \Psi_{1}^{-} \Psi_{2}^{+} \Psi_{3}^{+} \Lambda_{1}^{+} \Lambda_{2}^{+} \Lambda_{3}^{+},
$$


and the definition of the other sums $\Gamma_{j}^{(0)}$ is clear. Let

$$
\begin{aligned}
\gamma_{1}(k) & =\sum_{\substack{l\left|\mathcal{B}_{0}, m\right| \mathcal{P}_{0}, d \mid \mathcal{P}_{1} \\
d l m=k}} \mu(l) \lambda_{0}^{-}(m) \lambda_{1}^{+}(d), \\
\gamma_{j}(k) & =\sum_{\substack{l\left|\mathcal{B}_{0}, m\right| \mathcal{P}_{0}, d \mid \mathcal{P}_{1} \\
d l m=k}} \mu(l) \lambda_{0}^{+}(m) \lambda_{j}^{+}(d), \quad j=2,3 .
\end{aligned}
$$

Then by some routine arrangements we have

$$
\begin{aligned}
\Gamma_{1}^{(0)}= & \sum_{n \in \mathcal{F}^{*}} \sum_{\substack{l_{j}\left|\mathcal{B}_{0}, m_{j}\right| \mathcal{P}_{0}, d_{j} \mid \mathcal{P}_{1} \\
j=1,2,3}} \mu(\mathbf{l}) \lambda_{0}^{-}\left(m_{1}\right) \lambda_{0}^{+}\left(m_{2}\right) \lambda_{0}^{+}\left(m_{3}\right) \\
& \times \lambda_{1}^{+}\left(d_{1}\right) \lambda_{2}^{+}\left(d_{2}\right) \lambda_{3}^{+}\left(d_{3}\right) I(n ; \mathbf{l m d}) \\
= & \sum_{n \in \mathcal{F}^{*}} \sum_{\substack{k_{j} \leq \mathcal{B}_{0} D_{0} D_{j} \\
j=1,2,3}} \gamma_{1}\left(k_{1}\right) \gamma_{2}\left(k_{2}\right) \gamma_{3}\left(k_{3}\right) I(n ; \mathbf{k}) \\
= & \frac{\pi}{4} \sum_{n \in \mathcal{F}^{*}} \sum_{\substack { j \\
\begin{subarray}{c}{j=1,2,3 \\
j=1{ j \\
\begin{subarray} { c } { j = 1 , 2 , 3 \\
j = 1 } }\end{subarray}} \gamma_{1}\left(k_{1}\right) \gamma_{2}\left(k_{2}\right) \gamma_{3}\left(k_{3}\right) n^{1 / 2} \frac{\mathfrak{S}(n ; Q ; \mathbf{k})}{\varphi(\mathbf{k})} \\
& +\sum_{n \in \mathcal{F}^{*}} \sum_{\substack{k_{j} \leq \mathcal{B}_{0} D_{0} D_{j} \\
j=1,2,3}} \gamma_{1}\left(k_{1}\right) \gamma_{2}\left(k_{2}\right) \gamma_{3}\left(k_{3}\right) \mathfrak{R}(n ; Q ; \mathbf{k}) \\
= & \Gamma_{11}^{(0)}+\Gamma_{12}^{(0)} .
\end{aligned}
$$

Now Lemma 2 implies that

$$
\Gamma_{12}^{(0)} \ll X^{3} \log ^{-A} X .
$$

By Lemma 1 , for $l_{j}\left|\mathcal{B}_{0}, m_{j}\right| \mathcal{P}_{0}, d_{j} \mid \mathcal{P}_{j}, j=1,2,3$, we have

$$
\mathfrak{S}(n ; Q ; \mathbf{l m d})=8 \prod_{3 \leq p<Q_{0}}(1+t(p ; n ; \mathbf{l})) \prod_{Q_{0} \leq p<Q}(1+t(p ; n ; \mathbf{m})) .
$$

By (4.16) we get

$$
\Gamma_{11}^{(0)}=2 \pi \sum_{n \in \mathcal{F}^{*}} n^{1 / 2} \mathcal{J}(n) \mathcal{H}^{-}(n) \mathcal{G}_{1}^{+} \mathcal{G}_{2}^{+} \mathcal{G}_{3}^{+},
$$

where

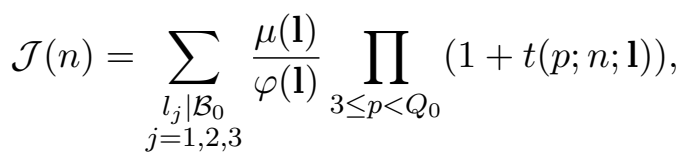




$$
\begin{aligned}
\mathcal{H}^{ \pm}(n) & =\sum_{\substack{m_{j} \mid \mathcal{P}_{0} \\
j=1,2,3}} \frac{\lambda_{0}^{ \pm}\left(m_{1}\right) \lambda_{0}^{+}\left(m_{2}\right) \lambda_{0}^{+}\left(m_{3}\right)}{\varphi(\mathbf{m})} \prod_{Q_{0} \leq p<Q}(1+t(p ; n ; \mathbf{m})), \\
\mathcal{G}_{j}^{ \pm} & =\sum_{d \mid \mathcal{P}_{j}} \frac{\lambda_{j}^{ \pm}(d)}{\varphi(d)}, \quad j=1,2,3 .
\end{aligned}
$$

By Lemma 3 it is easy to show that

$$
\mathcal{J}(n)=\prod_{3 \leq p<Q_{0}} \mathcal{V}_{p}(n)
$$

where

$$
\mathcal{V}_{p}(n)=\sum_{l_{1}, l_{2}, l_{3} \mid p} \frac{\mu(\mathbf{l})}{\varphi(\mathbf{l})}(1+t(p ; n ; \mathbf{l})) .
$$

By (3.15)-(3.18) of [16], for $n \in \mathcal{F}^{*}$ we have

$$
\begin{aligned}
& \mathcal{H}^{ \pm}(n)=\mathcal{H}_{0}(n)+O\left(\log ^{-2 A} X\right), \\
& (\log \log X)^{-9} \ll \mathcal{J}(n) \ll(\log \log X)^{9}, \\
& (\log \log X)^{-14} \ll \mathcal{H}_{0}(n) \ll(\log \log X)^{14}, \\
& \mathcal{G}_{j}^{ \pm} \ll \log X, \quad j=1,2,3,
\end{aligned}
$$

uniformly, where

$$
\mathcal{H}_{0}(n)=\prod_{\substack{\left.Q_{0} \leq p<Q \\ p, \mathcal{P}_{0}\right)=1}}\left(1+h_{0}(p)\right) \prod_{p \mid \mathcal{P}_{0}} \mathcal{V}_{p}(n) .
$$

By (4.18)-(4.21) we find that

$$
\Gamma_{11}^{(0)}=2 \pi \sum_{n \in \mathcal{F}^{*}} n^{1 / 2} \mathcal{J}(n) \mathcal{H}_{0}(n) \mathcal{G}_{1}^{+} \mathcal{G}_{2}^{+} \mathcal{G}_{3}^{+}+O\left(X^{3} \log ^{-A} X\right) .
$$

By (4.14)-(4.15) and (4.22) we get

$$
\Gamma_{1}^{(0)}=2 \pi \sum_{n \in \mathcal{F}^{*}} n^{1 / 2} \mathcal{J}(n) \mathcal{H}_{0}(n) \mathcal{G}_{1}^{+} \mathcal{G}_{2}^{+} \mathcal{G}_{3}^{+}+O\left(X^{3} \log ^{-A} X\right) .
$$

In a similar manner we obtain

$$
\begin{aligned}
\Gamma_{j}^{(0)} & =2 \pi \sum_{n \in \mathcal{F}^{*}} n^{1 / 2} \mathcal{J}(n) \mathcal{H}_{0}(n) \mathcal{G}_{1}^{+} \mathcal{G}_{2}^{+} \mathcal{G}_{3}^{+}+O\left(X^{3} \log ^{-A} X\right), \\
j=2,3,7, & \\
\Gamma_{4}^{(0)} & =2 \pi \sum_{n \in \mathcal{F}^{*}} n^{1 / 2} \mathcal{J}(n) \mathcal{H}_{0}(n) \mathcal{G}_{1}^{-} \mathcal{G}_{2}^{+} \mathcal{G}_{3}^{+}+O\left(X^{3} \log ^{-A} X\right), \\
\Gamma_{5}^{(0)} & =2 \pi \sum_{n \in \mathcal{F}^{*}} n^{1 / 2} \mathcal{J}(n) \mathcal{H}_{0}(n) \mathcal{G}_{1}^{+} \mathcal{G}_{2}^{-} \mathcal{G}_{3}^{+}+O\left(X^{3} \log ^{-A} X\right),
\end{aligned}
$$




$$
\Gamma_{6}^{(0)}=2 \pi \sum_{n \in \mathcal{F}^{*}} n^{1 / 2} \mathcal{J}(n) \mathcal{H}_{0}(n) \mathcal{G}_{1}^{+} \mathcal{G}_{2}^{+} \mathcal{G}_{3}^{-}+O\left(X^{3} \log ^{-A} X\right) .
$$

Now, (4.23)-(4.27) and (4.13) imply that

$$
\Gamma^{(0)} \geq 2 \pi \sum_{n \in \mathcal{F}^{*}} n^{1 / 2} \mathcal{J}(n) \mathcal{H}_{0}(n) \mathcal{G}+O\left(X^{3} \log ^{-A} X\right),
$$

where

$$
\mathcal{G}=\mathcal{G}_{1}^{-} \mathcal{G}_{2}^{+} \mathcal{G}_{3}^{+}+\mathcal{G}_{1}^{+} \mathcal{G}_{2}^{-} \mathcal{G}_{3}^{+}+\mathcal{G}_{1}^{+} \mathcal{G}_{2}^{+} \mathcal{G}_{3}^{-}-2 \mathcal{G}_{1}^{+} \mathcal{G}_{2}^{+} \mathcal{G}_{3}^{+} .
$$

By (2.2)-(2.3) in Lemma 4, we have

$$
\begin{array}{ll}
\mathcal{W}_{j} \leq \mathcal{G}_{j}^{+} \leq \mathcal{W}_{j}\left(F\left(s_{j}\right)+O\left(\log ^{-1 / 3} D_{j}\right)\right), & j=1,2,3, \\
\mathcal{W}_{j} \geq \mathcal{G}_{j}^{-} \geq \mathcal{W}_{j}\left(f\left(s_{j}\right)+O\left(\log ^{-1 / 3} D_{j}\right)\right), & j=1,2,3,
\end{array}
$$

where

$$
\mathcal{W}_{j}=\mathcal{W}\left(w_{j}\right)=\prod_{Q \leq p<w_{j}}\left(1-\frac{1}{p-1}\right) .
$$

Write $\mathcal{W}=\mathcal{W}_{1} \mathcal{W}_{2} \mathcal{W}_{3}$. Then by (4.29)-(4.31) we get

$$
\begin{aligned}
\mathcal{G} & =2\left(\mathcal{G}_{1}^{-}-\mathcal{G}_{1}^{+}\right) \mathcal{G}_{2}^{+} \mathcal{G}_{3}^{+}+\mathcal{G}_{1}^{+} \mathcal{G}_{2}^{+} \mathcal{G}_{3}^{-} \\
& \geq\left(2 f\left(s_{1}\right) F\left(s_{2}\right) F\left(s_{3}\right)-2 F\left(s_{1}\right) F\left(s_{2}\right) F\left(s_{3}\right)+f\left(s_{3}\right)+o(1)\right) \mathcal{W} \\
& \geq 0.99635 \mathcal{W},
\end{aligned}
$$

where Lemma 5 and numerical integration are employed. By (4.28) and (4.32) we obtain

$$
\Gamma^{(0)} \geq 0.99635 \cdot 2 \pi \sum_{n \in \mathcal{F}^{*}} n^{1 / 2} \mathcal{J}(n) \mathcal{H}_{0}(n) \mathcal{W}+O\left(X^{3} \log ^{-A} X\right) .
$$

- The upper bound for $\Gamma^{(1)}$. Write

$$
\gamma_{1}^{*}(k)=\sum_{\substack{l\left|\mathcal{B}_{0}, m\right| \mathcal{P}_{0}, d \mid \mathcal{P}_{1} \\ w_{1} \leq p<z_{1}, d l m p=k}} \mu(l) \lambda_{0}^{+}(m) \lambda_{1}^{+(p)}(d) g_{1}^{\prime}(p) .
$$

By (2.1) we have

$$
\begin{aligned}
\Gamma_{1}^{(1)} & =\sum_{n \in \mathcal{F}^{*}} \sum_{\substack{p_{1}^{2}+p_{2}^{2}+p_{3}^{2}=n \\
\left(p_{j}+2, Q_{j}\right)=1 \\
j=1,2,3}}(\log \mathbf{p}) g_{1}\left(p_{1}+2\right) \\
& =\sum_{n \in \mathcal{F}^{*}} \sum_{w_{1} \leq p<z_{1}} g_{1}^{\prime}(p) \sum_{\substack{p_{1}^{2}+p_{2}^{2}+p_{3}^{2}=n, p_{1}+2 \equiv 0(\bmod p) \\
\left(p_{j}+2, Q_{j}\right)=1 \\
j=1,2,3}} \log \mathbf{p}
\end{aligned}
$$




$$
\begin{aligned}
& =\sum_{n \in \mathcal{F}^{*}} \sum_{w_{1} \leq p<z_{1}} g_{1}^{\prime}(p) \sum_{\substack{p_{1}^{2}+p_{2}^{2}+p_{3}^{2}=n \\
p_{1}+2 \equiv 0(\bmod p)}} \log \mathbf{p} \\
& \times \Phi_{1} \Phi_{2} \Phi_{3} \Psi_{1} \Psi_{2} \Psi_{3} \Lambda_{1} \Lambda_{2} \Lambda_{3} \\
& \leq \sum_{n \in \mathcal{F}^{*}} \sum_{w_{1} \leq p<z_{1}} g_{1}^{\prime}(p) \sum_{\substack{p_{1}^{2}+p_{2}^{2}+p_{3}^{2}=n \\
p_{1}+2 \equiv 0(\bmod p)}} \log \mathbf{p} \\
& \times \Phi_{1} \Phi_{2} \Phi_{3} \Psi_{1}^{+} \Psi_{2}^{+} \Psi_{3}^{+} \Lambda_{1}^{+(p)} \Lambda_{2}^{+} \Lambda_{3}^{+} \\
& =\sum_{n \in \mathcal{F}^{*}} \sum_{\substack{k_{j} \leq \mathcal{B}_{0} D_{0} D_{j} \\
j=1,2,3}} \gamma_{1}^{*}\left(k_{1}\right) \gamma_{2}\left(k_{2}\right) \gamma_{3}\left(k_{3}\right) I(n ; \mathbf{k}) \\
& =\frac{\pi}{4} \sum_{n \in \mathcal{F}^{*}} \sum_{\substack{k_{j} \leq \mathcal{B}_{0} D_{0} D_{j} \\
j=1,2,3}} \gamma_{1}^{*}\left(k_{1}\right) \gamma_{2}\left(k_{2}\right) \gamma_{3}\left(k_{3}\right) n^{1 / 2} \frac{\mathfrak{S}(n ; Q ; \mathbf{k})}{\varphi(\mathbf{k})} \\
& +\sum_{n \in \mathcal{F}^{*}} \sum_{\substack{k_{j} \leq \mathcal{B}_{0} D_{0} D_{j} \\
j=1,2,3}} \gamma_{1}^{*}\left(k_{1}\right) \gamma_{2}\left(k_{2}\right) \gamma_{3}\left(k_{3}\right) \Re(n ; Q ; \mathbf{k}) \\
& =\Gamma_{11}^{(1)}+\Gamma_{12}^{(1)} \text {. }
\end{aligned}
$$

By Lemma 2 we find that

$$
\Gamma_{12}^{(1)} \ll X^{3} \log ^{-A} X .
$$

Similar to $\Gamma_{11}^{(0)}$, by (4.16) we obtain

$$
\Gamma_{11}^{(1)}=2 \pi \sum_{n \in \mathcal{F}^{*}} n^{1 / 2} \mathcal{J}(n) \mathcal{H}^{+}(n) \mathcal{G}_{1}^{+}\left(g_{1}^{\prime}\right) \mathcal{G}_{2}^{+} \mathcal{G}_{3}^{+},
$$

where

$$
\mathcal{G}_{j}^{+}\left(g_{j}^{\prime}\right)=\sum_{w_{j} \leq p<z_{j}} \frac{g_{j}^{\prime}(p)}{p-1} \sum_{d \mid \mathcal{P}_{j}} \frac{\lambda_{j}^{+(p)}(d)}{\varphi(d)}, \quad j=1,2,3 .
$$

By (2.3) we have

$$
\sum_{d \mid \mathcal{P}_{j}} \frac{\lambda_{j}^{+(p)}(d)}{\varphi(d)} \leq \mathcal{W}_{j}\left(F\left(\frac{\log D_{j} p^{-1}}{\log w_{j}}\right)+O\left(\log ^{-1 / 3} D_{j}\right)\right), \quad j=1,2,3 .
$$

By (4.37)-(4.38), the prime number theorem and summation by parts we find that

$$
\begin{aligned}
& \mathcal{G}_{j}^{+}\left(g_{j}^{\prime}\right) \leq(1+o(1)) \mathcal{W}_{j} \int_{1 / s_{j}}^{1-1 / s_{j}}\left(1-\frac{s_{j}}{s_{j}-1} t\right) \frac{F\left(s_{j}(1-t)\right)}{t} d t \\
& j=1,2,3 .
\end{aligned}
$$


By (4.18)-(4.21), (4.30) and (4.39) we get

$$
\begin{aligned}
\Gamma_{11}^{(1)} \leq & (1+o(1)) C_{1} \cdot 2 \pi \sum_{n \in \mathcal{F}^{*}} n^{1 / 2} \mathcal{J}(n) \mathcal{H}_{0}(n) \mathcal{W} \\
& +O\left(X^{3} \log ^{-A} X\right),
\end{aligned}
$$

where

$$
C_{1}=F(5) F(6) \int_{1 / 5}^{4 / 5}\left(1-\frac{5 t}{4}\right) \frac{F(5(1-t))}{t} d t .
$$

By (4.35), (4.40)-(4.41), Lemma 5 and numerical integration, we obtain

$$
\Gamma_{1}^{(1)} \leq 0.77133 \cdot 2 \pi \sum_{n \in \mathcal{F}^{*}} n^{1 / 2} \mathcal{J}(n) \mathcal{H}_{0}(n) \mathcal{W}+O\left(X^{3} \log ^{-A} X\right) .
$$

By the same arguments we get

$$
\begin{aligned}
\Gamma_{2}^{(1)} \leq & (1+o(1)) C_{1} \cdot 2 \pi \sum_{n \in \mathcal{F}^{*}} n^{1 / 2} \mathcal{J}(n) \mathcal{H}_{0}(n) \mathcal{W} \\
& +O\left(X^{3} \log ^{-A} X\right) \\
\leq & 0.77133 \cdot 2 \pi \sum_{n \in \mathcal{F}^{*}} n^{1 / 2} \mathcal{J}(n) \mathcal{H}_{0}(n) \mathcal{W}+O\left(X^{3} \log ^{-A} X\right), \\
\Gamma_{3}^{(1)} \leq & (1+o(1)) C_{3} \cdot 2 \pi \sum_{n \in \mathcal{F}^{*}} n^{1 / 2} \mathcal{J}(n) \mathcal{H}_{0}(n) \mathcal{W} \\
& +O\left(X^{3} \log ^{-A} X\right) \\
\leq & 0.89182 \cdot 2 \pi \sum_{n \in \mathcal{F}^{*}} n^{1 / 2} \mathcal{J}(n) \mathcal{H}_{0}(n) \mathcal{W}+O\left(X^{3} \log ^{-A} X\right),
\end{aligned}
$$

where

$$
C_{3}=F(5) F(5) \int_{1 / 6}^{5 / 6}\left(1-\frac{6 t}{5}\right) \frac{F(6(1-t))}{t} d t .
$$

By (4.42)-(4.44) we find that

$$
\begin{aligned}
\Gamma^{(1)} & =\sum_{j=1}^{3} \theta_{j} \Gamma_{j}^{(1)} \\
& \leq 0.98947 \cdot 2 \pi \sum_{n \in \mathcal{F}^{*}} n^{1 / 2} \mathcal{J}(n) \mathcal{H}_{0}(n) \mathcal{W}+O\left(X^{3} \log ^{-A} X\right) .
\end{aligned}
$$

By (4.1), (4.33) and (4.45) we get

$$
\begin{aligned}
\Gamma & =\Gamma^{(0)}-\Gamma^{(1)} \\
& \geq 0.006 \cdot 2 \pi \sum_{n \in \mathcal{F}^{*}} n^{1 / 2} \mathcal{J}(n) \mathcal{H}_{0}(n) \mathcal{W}+O\left(X^{3} \log ^{-A} X\right) .
\end{aligned}
$$


C) Proof of Proposition 1. Upon comparing (4.12) and (4.46) we obtain

$$
\mathcal{Y}^{*}(N)=\sum_{n \in \mathcal{F}^{*}} 1 \ll X^{2} \log ^{5-A} X,
$$

where (4.19)-(4.20) and the bound

$$
\mathcal{W} \gg \frac{\log ^{3} \log X}{\log ^{3} X},
$$

a consequence of Mertens' product formula, have been used.

By (4.47) and the bound (see [7, Chapter 0])

$$
\mathcal{Y}(N)-\mathcal{Y}^{*}(N) \ll X^{2}(\log X)^{-A \log A-A-1},
$$

we get $\mathcal{Y}(N) \ll X^{2} \log ^{5-A} X$, and Proposition 1 follows.

5. Proof of the theorems. In this paper we present only the proof of Theorem 1. From Propositions 2 and $2^{\prime}$, Theorems 2 and $2^{\prime}$ follow by similar but simpler arguments (see [15] for the details). Let

$$
\begin{aligned}
\mathfrak{A} & =\left\{p: p \leq n^{1 / 2}, p \equiv 11(\bmod 30), p+2=P_{2}\right\}, \\
\mathfrak{A}^{\prime} & =\left\{p: p \leq n^{1 / 2}, p \equiv 17(\bmod 30), p+2=P_{2}\right\} .
\end{aligned}
$$

By Chen's argument in [2], we have

$$
\begin{array}{r}
|\mathfrak{A}| \gg n^{1 / 2} \log ^{-2} n, \\
\left|\mathfrak{A}^{\prime}\right| \gg n^{1 / 2} \log ^{-2} n .
\end{array}
$$

CASE $1: n \not \equiv 2(\bmod 5)$. Let

$$
\mathscr{A}=\left\{n-p_{1}^{2}-p_{2}^{2}: p_{1}, p_{2} \in \mathfrak{A}\right\}, \quad r^{\prime}(k)=\sum_{\substack{p_{1}^{2}+p_{2}^{2}=k \\ p_{1}, p_{2} \in \mathfrak{A}}} 1, \quad r(k)=\sum_{m_{1}^{2}+m_{2}^{2}=k} 1 .
$$

Then we have

$$
\sum_{\substack{k \in \mathscr{A} \\ r^{5}(k)>\log ^{5} n}} 1 \leq \frac{1}{\log ^{5} n} \sum_{k \leq n} r^{\prime}(k) \leq \frac{1}{\log ^{5} n} \sum_{k \leq n} r(k) \ll \frac{n}{\log ^{5} n} .
$$

By (5.1), (5.3) and Dirichlet's pigeon hole principle we know that $\mathscr{A}$ contains $\gg n \log ^{-9} n$ distinct integers $k$ satisfying $k \equiv 3(\bmod 24)$ and $k \not \equiv 0(\bmod 5)$, and Theorem 1 follows from Proposition 1.

CASE $2: n \equiv 2(\bmod 5)$. Letting

$$
\mathscr{A}^{\prime}=\left\{n-p_{1}^{2}-p_{2}^{2}: p_{1} \in \mathfrak{A}, p_{2} \in \mathfrak{A}^{\prime}\right\},
$$

and then proceeding as in Case 1, we get the proof of Theorem 1.

Acknowledgements. The authors would like to thank Professor D. I. Tolev for his assistance in the course of studying [16], and the referee for his/her patience and time in refereeing this paper. 
This project was supported by the National Natural Science Foundation of China (grant no. 10171060, 10171076 and 10471104) .

\section{References}

[1] J. Brüdern and E. Fouvry, Lagrange's Four Squares Theorem with almost prime variables, J. Reine Angew. Math. 454 (1994), 59-96.

[2] J. R. Chen, On the representation of a large even integer as the sum of a prime and the product of at most two primes, Sci. Sinica 16 (1973), 157-176.

[3] B. Green and T. Tao, Restriction theory of the Selberg sieve with applications, J. Théor. Nombres Bordeaux 18 (2006), 147-182.

[4] - - - The primes contain arbitrarily long arithmetic progressions, Ann. of Math. 167 (2008), 481-547.

[5] H. Halberstam, D. R. Heath-Brown and H. E. Richert, Almost-primes in short intervals, in: Recent Progress in Analytic Number Theory, Academic Press, London, 1981, 69-101.

[6] H. Halberstam and H. E. Richert, Sieve Methods, Academic Press, London, 1974.

[7] R. R. Hall and G. Tenenbaum, Divisors, Cambridge Univ. Press, 1988.

[8] D. R. Heath-Brown, Three primes and an almost-prime in arithmetic progression, J. London Math. Soc. (2) 23 (1981), 396-414.

[9] L. K. Hua, Some results in additive prime number theory, Quart. J. Math. Oxford 9 (1938), 68-80.

[10] H. Iwaniec, Rosser's sieve, Acta Arith. 36 (1980), 171-202.

[11] - A new form of the error term in the linear sieve, ibid. 36 (1980), 307-320.

[12] T. P. Peneva, On the ternary Goldbach problem with primes $p_{i}$ such that $p_{i}+2$ are almost-primes, Acta Math. Hungar. 86 (2000), 305-318.

[13] T. P. Peneva and D. I. Tolev, An additive problem with primes and almost-primes, Acta Arith. 83 (1998), 155-169.

[14] D. I. Tolev, Arithmetic progressions of prime-almost-prime twins, ibid. 88 (1999), 67-98.

[15] —, Representations of large integers as a sum of two primes of special type, in: Algebraic Number Theory and Diophantine Analysis, de Gruyter, 2000, 485-495.

[16] - Additive problems with prime numbers of special type, Acta Arith. 96 (2000), 53-88; Corrigendum, ibid. 105 (2002), 205.

[17] J. G. van der Corput, Über Summen von Primzahlen und Primzahlquadraten, Math. Ann. 116 (1939), 1-50.

[18] I. M. Vinogradow, Representation of an odd number as a sum of three primes, C. R. (Doklady) Acad. Sci. URSS 15 (1937), 291-294.

Yingchun Cai

Department of Mathematics

Tongji University

Shanghai, 200092, P.R. China

E-mail: yingchuncai@tongji.edu.cn
Minggao Lu

Department of Mathematics

Shanghai University

Shanghai, 200436, P.R. China

E-mail: lumg0202@online.sh.cn

Received on 27.1.2009

and in revised form on 10.4.2009 